\title{
Gedanken eines Oberarztes zu den Arbeitszeiten der Ärzte im Spital
}

F. Kurth

Korrespondenz: Dr. med. F. Kurth Spital Dornach CH-4143 Dornach
Auf den Beitrag von Herrn R. Gonzenbach [1] ist meines Erachtens eine Replik eines Vertreters meiner Generation nötig.

Ich habe 1990, also etwa 40 Jahre nach Herrn Gonzenbach, Examen gemacht. Das liegt so lange zurück, dass ich Zeit hatte, ein wenig Erfahrung im Beruf zu sammeln, das ist so wenig lange her, dass ich mit den inzwischen stattgefundenen Veränderungen Schritt halten konnte. Allerdings - aber das ist aus meiner Sicht kein Manko - bin ich zu jung, um meine Erfahrungen im Berufsleben mit Verhaltensregeln im Krieg zu korrelieren, wie das Herr Gonzenbach zu tun offenbar für wichtig hält.

Als Assistenzarzt habe ich früher häufig Wochen mit mehr als 110 Stunden Arbeitszeit geschafft und habe inzwischen als Oberarzt eine Sollarbeitszeit von 48 Stunden pro Woche. Ich meine daher, beide Arbeitswelten aus eigener Erfahrung beurteilen zu können.

Herr Gonzenbach kritisiert die wegen der Arbeitszeitreduktion nötigen häufigeren Schichtwechsel und weist auf die dadurch entstehende Fehlerquelle hin. Dieser Hinweis erfolgt völlig zu Recht, hier besteht eine Problematik, deren Lösung sich zurzeit viele Personen intensiv und vor allem konstruktiv widmen.

In seinem Beitrag geht Herr Gonzenbach aber davon aus, dass im Spital der tägliche DreiSchichten-Betrieb eingeführt werden müsste. Diese Annahme ist jedoch falsch und entspricht nicht der gelebten Realität. Es wird in den allermeisten Spitälern versucht, in den Abteilungen, die wegen kurzer Liegedauer bzw. täglich wechselnder Patienten weniger auf Kontinuität im Assistenzarztbereich angewiesen sind (Ambulanz, Intensivstation), die häufigen Assistentenwechsel zu konzentrieren.

Die Stationen mit längerer Aufenthaltsdauer werden aber weiterhin einem zuständigen Stationsarzt zugeordnet. Dieser ist zugegebenermassen nicht 7 Tage die Woche jeweils 24 Stunden pro Tag anwesend, aber das war auch vor 20 , 30 und 40 Jahren nicht so.

Diese Assistenzärzte arbeiten 5 Tage pro Woche je 10 Stunden und decken damit die kontinuierliche Patientenbetreuung ab, sie können sowohl an Rapporten teilnehmen als auch ope- rieren und visitieren, so wie das schon immer war. Die von Herrn Gonzenbach behauptete Zahl von 5 verschiedenen zuständigen Ärzten pro Woche ist also stark übertrieben. Als Informationsträger, Hauptkoordinator und Ansprechperson ist auf der Normalstation auch heutzutage ein Arzt zuständig.

Auch zu Zeiten von Herrn Gonzenbach war es in einer Woche durchaus üblich, dass von Montag bis Freitag tagsüber ein einzelner Assistent zuständig war, in der Nacht mal dieser eine, mal aber auch ein anderer Arzt Dienst hatte und dass auch am Wochenende nicht immer nur der eine einzige Arzt arbeitete, sondern, wenn dieser doch mal ein freies Wochenende hatte, einer seiner Kollegen Dienst tat. Auch Ferien wird es in dieser alten Zeit gegeben haben, und wenn ich nicht irre, auch Militärdienst, sogar Krankheit der Ärzte und wissenschaftliche Urlaube. So wird also auch vor vielen Jahren ein Patient, der 7 Tage hospitalisiert war (und damals waren die allermeisten viel, viel länger im Spital), seine 3 Assistenzarztgesichter pro Woche gesehen haben. Der Unterschied zu einer Normalstation von heutzutage ist nicht wirklich gross. Ein wesentlicher Teil der Argumentation von Herrn Gonzenbach hat somit keine reale Grundlage.

Ich möchte überhaupt nicht bestreiten, dass der Wechsel von Assistenzärzten Probleme aufwirft, aber neu sind diese gar nicht, da es immer schon Wechsel gab. Es brauchte schon immer Lösungen für wechselnde Nacht- und Wochenenddiensthabende, nur jetzt, da diese Wechsel häufiger und regelhaft auftreten, muss man sich ernsthafter und umfassender um Lösungen bemühen.

Hier nur auf die alte Zeit zu verweisen, die tatsächlich vergangen ist, löst keines der anstehenden Probleme. Schon gar nicht hilfreich ist eine unnötige Dramatisierung der Veränderungen.

Der Tatsache, dass Herr Gonzenbach sich an keinen Fehler durch Übermüdung während seiner gesamten Arbeitszeit als Chirurg erinnern kann, liegt wohl eher ein Nichtwahrnehmen zugrunde, als dass tatsächlich nie Fehler aufgetreten wären. In zwei kürzlich im «New England Journal of Medicine» erschienenen Beiträgen [2, 3] wird aufgezeigt, dass es die Fehler durch Über- 
müdung und Überarbeitung sehr wohl gibt und sich diese durch Arbeitszeitverkürzung auch vermeiden lassen. Hier sollten wir Ärzte uns vom Bild des auch bei stärkster Belastung und trotz Schlafmankos fehlerlos funktionierenden «Halbgottes in Weiss» endlich lösen.

Herr Gonzenbach spricht vom angeblich umgehängten Mäntelchen der Überforderung. Aber die Überforderung war und ist objektiv, sie $\mathrm{zu}$ beseitigen bringt Vorteile, ganz sicher auch für die Patienten.

Das Mäntelchen der aufopfernden Liebe zu den Patienten als alleinige Triebfeder der ärztlichen Tätigkeit, das sich Ärzte früherer Generationen gerne umlegten, sollte bitte auch abgeworfen werden.

Herr Gonzenbach sieht zwar, dass es veränderte Lebenspläne von jüngeren Männern und Frauen auch in Ärztekreisen gibt. Aber er beklagt dann doch nur eine nicht mehr zu ändernde moderne Lebensweise mit neuen Freiheiten, die eine menschliche Beziehung zwischen Arzt und Patient verhindere.

Dass Frauen nicht mehr nur als Hausfrau und Mutter eine Lebensberechtigung haben wollen, sondern auch von der Möglichkeit Gebrauch machen, einen anderen Beruf ausüben zu können, ist eine Tatsache, die aus meiner Sicht nur positiv zu werten ist. So haben aber weder diese Frauen noch deren Männer, wenn diese auch berufstätig sind, eine aufopferungsvolle Stütze im Hintergrund, wie das die Männer der Generation Gonzenbach noch hatten, die ihren Frauen noch nicht mal das Wahlrecht zugestehen wollten. Diese Frauen machten ja oft erst eine Karriere dieser Männer, die Gründung einer Familie trotz extremer Arbeitsbelastung und die Organisation von sozialen Kontakten möglich.

Daraus ergeben sich die heute veränderten Lebenspläne, die nicht nur ein lustvolles Nachgeben gegenüber verlockender Freiheit darstellen.

Herr Gonzenbach, der Teilzeitkräfte nur akzeptieren wollte, wenn sie im Wechsel 3 Monate $100 \%$ und dann 3 Monate gar nicht arbeiteten, hatte sich offenbar sehr wenig Gedanken gemacht, wie denn in der Phase mit 100\% Arbeitszeit ein Familienleben organisiert werden soll, und er hätte vielleicht etwas mehr Phanta- sie aufbringen müssen, um zu probieren, ob sich nicht die Arbeitsbedingungen an Menschen, statt nur die Menschen sich an starre Arbeitsbedingungen anpassen könnten.

Dass Ärzte, die nicht bis zur Erschöpfung arbeiten, auch viel mehr Reserven haben, um anspruchsvolle und anstrengende Patienten zu betreuen, dass Teilzeitarbeitende, wenn ihnen nicht sämtliche Karrieremöglichkeiten beschnitten werden, oft viel motivierter und auch geduldiger und zugewandter sind als Vollzeitkräfte, sollte von viel mehr Chefs bedacht werden.

Die zum Abschluss wie eine Tatsache postulierte These, dass Arbeit nur soviel Befriedigung schenkt, wie wir an Anstrengung und Mühen bereit sind einzubringen, ist keinesfalls bewiesen, ich halte sie nicht für richtig. Sie führt ins Absurde, wenn man sie weiterdenkt: gibt es durch grenzenlosen Einsatz und unendliche Mühe etwa absolute Befriedigung?

Die Grenze zum Workaholic wird bei dieser Prämisse schnell überschritten.

Ist nicht eine Tätigkeit wirklich befriedigend, die man ausgeruht beginnen und noch vor Erreichen der Erschöpfungsgrenze wieder beenden kann? Befriedigt nicht vielmehr eine anspruchsvolle Arbeit, die auch Zeit lässt für die intensive Beschäftigung mit der Familie, mit Themen ausserhalb der Spitalmauern und für soziale Kontakte, deren Erfahrung ja auch immer wieder zurückfliessen in einen derart lebensnahen Beruf wie den des Arztes?

Mein Arbeitsleben lehrt mich: Solch eine Arbeit ist sehr wohl befriedigend auch und gerade wenn ich sie «nur» 50 Stunden pro Woche oder gar noch weniger lange ausübe.

\section{Literatur}

1 Gonzenbach R. Arbeitszeiten der Assistenten (und Oberärzte?) im Spital. Schweiz Ärztezeitung 2005;86(11):696-7.

2 Lockley SW, Cronin JW, Evans EE, Cade BE, Lee CJ, Landrigan CP, et al. Effects of reducing interns' weekly work hours on sleep and attentional failures. N Engl J Med 2004;351:1829-37.

3 Landrigan CP, Rothschild JM, Cronin JW, Kaushal R, Burdick E, Katz JT, et al. Effects of reducing interns' weekly work hours on serious medical errors in intensive care units. N Engl J Med 2004; 351:1838-48. 